\title{
Prevalence and determinants of mental distress among university students in Ethiopia: a systematic review protocol
}

Berihun Assefa Dachew ${ }^{1 *} \mathbb{D}$, Berhanu Boru Biffu ${ }^{2}$, Bewket Tadesse Tiruneh ${ }^{2}$, Degefaye Zelalem Anlay ${ }^{2}$, Meseret Adugna Wassie ${ }^{3}$ and Kim Betts ${ }^{4}$

\begin{abstract}
Background: Mental distress is an important public health problem and becoming a common health problem among university students. This systematic review and meta-analysis will provide the pooled prevalence of mental distress and identify determinant factors associated with mental distress among university students in Ethiopia.

Method: A systematic search of PubMed, EMBASE, and PsycINFO databases will be conducted. In addition, we will search grey literature resources such as a database/website of dissertations and theses, WHO websites, and websites of professional bodies. Reference lists of the selected articles will also be searched for additional articles. All observational studies reporting the prevalence of mental distress and/or associated factors among university students in Ethiopia will be included. Pooled prevalence with $95 \%$ confidence interval $(95 \% \mathrm{Cl})$ will be calculated using randomeffects and quality-effects models. Subgroup and sensitivity analyses will be performed. Heterogeneity between studies and evidence of publication bias will also be assessed.

Discussion: The proposed systematic review and meta-analysis will provide a pooled prevalence of mental distress and associated factors to assist policy-makers and programme managers in developing evidence-based mental health promotion and prevention programmes in university/college settings.
\end{abstract}

Systematic review registration: PROSPERO CRD42017067223

Keywords: Prevalence, Mental distress, University students, Ethiopia

\section{Background}

Mental distress is a mental health problem characterized with a range of depressive, anxiety, or somatic symptoms such as headache, backache, and sleep problems $[1,2]$. A person with mental distress may also present with confused emotions and hallucinations [2, 3]. The term is used when it has not been possible to obtain a specific diagnosis of mental disorder [3]. Mental distress affects a substantial proportion of the world population and is prevalent in universities worldwide [2, 4-9].

\footnotetext{
* Correspondence: berihunassefa21@gmail.com

${ }^{1}$ Department of Epidemiology and Biostatistics, Institute of Public Health, College of Medicine and Health Sciences, University of Gondar, Gondar, Ethiopia

Full list of author information is available at the end of the article
}

There are various factors that could contribute to mental distress among university students. The experience of new lifestyles, new roommates and friends, exposure to new cultures and environments, loss of traditional adult supervisors, and low social support are some of these factors $[6,10-12]$. In addition, academic stressors, peer pressure, and financial distress have been recognized as risk factors for developing mental distress among students $[5,13]$. However, many students do not seek help for their problems. Inability to manage these conditions early will lead to adverse impacts on their academic achievement [14, 15]. More importantly, mental distress is associated with an increased risk of substance use [16, 17] and suicidal behaviours [18-20].

In Ethiopia, mental and substance use disorders are common public health problems responsible for about

(c) The Author(s). 2019 Open Access This article is distributed under the terms of the Creative Commons Attribution 4.0 International License (http://creativecommons.org/licenses/by/4.0/), which permits unrestricted use, distribution, and reproduction in any medium, provided you give appropriate credit to the original author(s) and the source, provide a link to the Creative Commons license, and indicate if changes were made. The Creative Commons Public Domain Dedication waiver (http://creativecommons.org/publicdomain/zero/1.0/) applies to the data made available in this article, unless otherwise stated. 
1897 disability-adjusted life years (DALYs) per 100,000 population [21]. Studies have shown that mental distress prevalence among university students in Ethiopia varies from 21.6 to $63.1 \%$ [5, 22-26]. Although several individual studies have reported the prevalence of mental distress and associated factors among university students in Ethiopia, to our knowledge there is no published systematic review and meta-analysis that shows pooled estimates of mental distress and its associated factors. Having a pooled prevalence of mental distress and identifying the associated factors would help policy-makers and programme managers in developing evidence-based mental health promotion and diseases prevention programmes. Therefore, the objective of this systematic review and meta-analysis is to review the existing literature, with the aim of quantifying the burden of mental distress and identifying factors associated with mental distress among university students in Ethiopia.

\section{Methods}

This protocol adheres to the Preferred Reporting Items for Systematic Reviews and Meta-analysis Protocols (PRISMA-P) [27] (Additional file 1). The review protocol has been registered in the International Prospective Register of Systematic Reviews (PROSPERO) (ref CRD42017067223).

\section{Data sources and search strategies}

A systematic search of PubMed (from its inception to present), EMBASE (1947 to present), and PsycINFO (1967 to present) databases will be conducted in accordance with a detailed search strategy. The search comprises both Medical Subject Headings (MeSH) and free text words (title and abstract word searches). We will use the following search terms: "Mental Disorders"[Mesh], "Somatoform Disorders/diagnosis"[Mesh]) in combination with "student"", "university student", "college student"*, and "Ethiopian*". The full electronic search strategies are included in the supplementary information (Additional file 2). In addition, we will search grey literature resources such as a database/website of dissertations and theses, WHO websites, and websites of professional bodies (e.g. mental health professional's association and mental health charities). The reference lists of included studies will be manually searched for additional eligible articles. We will contact authors when additional information is required.

\section{Inclusion and exclusion criteria}

All observational studies reporting the prevalence of mental distress and/or factors associated with mental distress among university students will be included. An article will be included if it meets the following criteria: (1) conducted solely or partly among university or college students, (2) reported the prevalence of mental distress using standardized instruments or questionnaires (such as Beck's Depression Inventory, Patient Health Questionnaire-9, Self-reporting Questionnaire-20, or clinical interviews) or provided sufficient information to calculate prevalence, and (3) published in English. When studies include a sample partly composed of university/ college students, we will extract required information to calculate prevalence. Conference abstracts, letters to editors, review, and commentary articles and studies where the participants are not human will be excluded. Non-English language studies will also be excluded as we do not have resources (to pay or find volunteers) to translate the data.

\section{Selection of studies for inclusion in the review}

Titles and abstracts of studies retrieved from each database search will be stored and managed in an EndNote reference manager. Two review authors (BAD and MAW) will independently review the titles and abstracts of all studies and disagreements will be solved by discussion.

\section{Data extraction and management}

Using a standardized data extraction form, two review authors (BAD and MAW) will independently extract data from eligible studies including first author's last name, year of publication, study location, sample size, number of events, data on prevalence of mental distress, ascertainment of outcome, risk or protective factors examined in each study together with their respective odds ratio (OR) and 95\% confidence intervals (CI), and information for assessment of the risk of bias.

\section{Quality assessment}

Two review authors (BAD and MAW) will independently assess the quality of all included studies using the Newcastle-Ottawa Quality Assessment tool adapted for cross-sectional studies [28]. Discrepancies will be resolved by a third reviewer (BTT) if necessary. The tool takes into account the selection of participants, comparability, and assessment of outcome. For quantitative analysis, quality scores will be assigned by dividing each score by the score of the highest scoring study in the group.

\section{Data synthesis}

The pooled prevalence (proportion) of mental distress and the pooled odds ratios (OR) of identified factors associated with mental distress with $95 \%$ CI will be calculated using random-effects [29] and quality-effects models [30]. Quality-effects meta-analysis will be used to examine how the quality of each study changed the pooled estimate compared with the results from 
random-effects meta-analysis. This analysis incorporates the quality score of each study in the calculation of the study weight, which is a robust and innovative technique to help minimize the estimator variance and account for subjectivity in quality assessment. Heterogeneity between the studies will be assessed using both Cochrane's Q statistic and the $I^{2}$ statistics. $I^{2}$ value greater than $50 \%$ will be considered as indicative of substantial heterogeneity [31]. Funnel plots will be used to assess publication bias [32]. Double arcsine transformation will be used if there is variance instability.

Subgroup analyses will be performed to explore the sources of heterogeneity attributed to gender, sample size, year of publication, study quality, year of study, variation in thresholds, and assessment methods/instruments. Sensitivity analysis will be performed by excluding each study one by one and calculating a pooled estimate for the remainder of the studies. All statistical analyses will be performed using MetaXL version 5.3 and STATA14 Metaprop package.

\section{Presenting and reporting the results}

A flow diagram will be included to outline the study selection process step by step, and a rationale provided for excluded studies. The characteristics and quality assessment of the included studies will be presented in tables. Pooled estimates will be presented using forest plots.

\section{Discussion}

This will be the first systematic review and meta-analysis that will determine the pooled prevalence of mental distress among university students in Ethiopia. It will also identify factors associated with mental distress among students. By identifying the risk and protective factors of mental distress, the findings may help in developing evidence-based mental health promotion and diseases prevention programmes targeting university students.

\section{Potential limitations}

It is anticipated that publication bias may pose as a limitation for this review. There is also a possibility of reporting and response set bias.

\section{Additional files}

Additional file 1: PRISMA-P (Preferred Reporting Items for Systematic review and Meta-Analysis Protocols) 2015 checklist: recommended items to address in a systematic review protocol. (DOCX $15 \mathrm{~kb}$ )

Additional file 2: Example search used for identification of articles on the PubMed database. (DOCX $12 \mathrm{~kb}$ )

\section{Abbreviations}

95\% Cl: 95\% confidence interval; DALYs: Disability-adjusted life years; MeSH: Medical Subject Headings; PRISMA-P: Preferred Reporting Items for Systematic Reviews and Meta-analysis; WHO: World Health Organization
Acknowledgements

None.

Funding

The authors received no specific funding for this work.

Availability of data and materials

Not applicable.

\section{Authors' contributions}

BAD conceived and designed the study. BAD and MAW designed the search strategy. BAD drafted the first manuscript. BBB, BTT, DZA, MAW, and KB critically reviewed the protocol. All authors read and approved the final draft of the protocol.

Ethics approval and consent to participate

Not applicable.

\section{Consent for publication}

Not applicable.

\section{Competing interests}

The authors declare that they have no competing interests.

\section{Publisher's Note}

Springer Nature remains neutral with regard to jurisdictional claims in published maps and institutional affiliations.

\section{Author details}

${ }^{1}$ Department of Epidemiology and Biostatistics, Institute of Public Health, College of Medicine and Health Sciences, University of Gondar, Gondar, Ethiopia. ${ }^{2}$ School of Nursing, College of Medicine and Health Sciences, University of Gondar, Gondar, Ethiopia. ${ }^{3}$ Department of Health Informatics, Teda Health Science College, Gondar, Ethiopia. ${ }^{4}$ School of Public Health, Curtin University, Perth, Australia.

Received: 8 January 2018 Accepted: 28 January 2019

Published online: 07 February 2019

\section{References}

1. de Waal MW, Arnold IA, Spinhoven P, Eekhof JA, van Hemert AM. The reporting of specific physical symptoms for mental distress in general practice. J Psychosom Res. 2005:59(2):89-95.

2. Rocha SV, de Almeida MM, de Araujo TM, Virtuoso JS Jr. Prevalence of common mental disorders among the residents of urban areas in Feira de Santana, Bahia. Rev Bras Epidemiol. 2010;13(4):630-40.

3. Giang KB, Dzung TV, Kullgren G, Allebeck P. Prevalence of mental distress and use of health services in a rural district in Vietnam. Glob Health Action. 2010;3. https://doi.org/10.3402/gha.v3i0.2025.

4. Patel V, Kleinman A. Poverty and common mental disorders in developing countries. Bull World Health Organ. 2003;81(8):609-15.

5. Dachew BA, Azale Bisetegn T, Berhe Gebremariam R. Prevalence of mental distress and associated factors among undergraduate students of University of Gondar, Northwest Ethiopia: a cross-sectional institutional based study. PLoS One. 2015;10(3):e0119464

6. Mosley TH Jr, Perrin SG, Neral SM, Dubbert PM, Grothues CA, Pinto BM. Stress, coping, and well-being among third-year medical students. Acad Med. 1994;69(9):765-7.

7. Sidik SM, Rampal L, Kaneson N. Prevalence of emotional disorders among medical students in a Malaysian university. Asia Pac Fam Med. 2003;2:213-7.

8. Stallman HM. Prevalence of psychological distress in university students-implications for service delivery. Aust Fam Physician. 2008;37(8):673-7.

9. Lima MC, Domingues Mde S, Cerqueira AT. Prevalence and risk factors of common mental disorders among medical students. Rev Saude Publica. 2006:40(6):1035-41.

10. Storrie K, Ahern K, Tuckett A. A systematic review: students with mental health problems--a growing problem. Int J Nurs Pract. 2010;16(1):1-6.

11. Yussuf AD, Issa BA, Ajiboye PO, Buhari Ol. The correlates of stress, coping styles and psychiatric morbidity in the first year of medical education at a Nigerian University. Afr J Psychiatry. 2013;16(3):206-15. 
12. Al-Busaidi Z, Bhargava K, Al-Ismaily A, Al-Lawati H, Al-Kindi R, Al-Shafaee M, Al-Maniri A. Prevalence of depressive symptoms among university students in Oman. Oman Med J. 2011;26(4):235-9.

13. Sreeramareddy CT, Shankar PR, Binu VS, Mukhopadhyay C, Ray B, Menezes RG. Psychological morbidity, sources of stress and coping strategies among undergraduate medical students of Nepal. BMC Med Educ. 2007;7:26.

14. Kessler RC, Foster CL, Saunders WB, Stang PE. Social consequences of psychiatric disorders, I: educational attainment. Am J Psychiatry. 1995;152(7): 1026-32.

15. Sijtsema JJ, Verboom CE, Penninx BW, Verhulst FC, Ormel J. Psychopathology and academic performance, social well-being, and social preference at school: the TRAlLS study. Child Psychiatry Hum Dev. 2014; 45(3):273-84.

16. Kingston REF, Marel C, Mills KL. A systematic review of the prevalence of comorbid mental health disorders in people presenting for substance use treatment in Australia. Drug Alcohol Rev. 2017;36(4):527-39.

17. Swendsen J, Conway KP, Degenhardt L, Glantz M, Jin R, Merikangas KR, Sampson N, Kessler RC. Mental disorders as risk factors for substance use, abuse and dependence: results from the 10-year follow-up of the National Comorbidity Survey. Addiction. 2010;105(6):1117-28.

18. Dvorak RD, Lamis DA, Malone PS. Alcohol use, depressive symptoms, and impulsivity as risk factors for suicide proneness among college students. J Affect Disord. 2013;149(1-3):326-34

19. Garlow SJ, Rosenberg J, Moore JD, Haas AP, Koestner B, Hendin H, Nemeroff CB. Depression, desperation, and suicidal ideation in college students: results from the American Foundation for Suicide Prevention College Screening Project at Emory University. Depress Anxiety. 2008;25(6):482-8.

20. Izadinia N, Amiri M, Jahromi R, Hamidi S. A study of relationship between suicidal ideas, depression, anxiety, resiliency, daily stresses and mental health among Tehran university students. Procedia Soc Behav Sci. 2010;5: 1615-9.

21. Institute for Health Metrics and Evaluation (IHME). GBD compare data visualization. Seattle: IHME, University of Washington; 2016. Available from http://vizhub.healthdata.org/gbd-compare. Accessed 15 May 2017.

22. Alem A, Araya M, Melaku Z, Wendimagegn D, Abdulahi A. Mental distress in medical students of Addis Ababa University. Ethiop Med J. 2005;43(3):159-66.

23. Tesfaye A. Prevalence and correlates of mental distress among regular undergraduate students of Hawassa University: a cross sectional survey. East Afr J Public Health. 2009:6(1):85-94.

24. Dessie Y, Ebrahim J, Awoke T. Mental distress among university students in Ethiopia: a cross sectional survey. Pan Afr Med J. 2013;15:95.

25. Melese B, Bayu B, Wondwossen F, Tilahun K, Lema S, Ayehu M, Loha E. Prevalence of mental distress and associated factors among Hawassa University medical students, Southern Ethiopia: a cross-sectional study. BMC Res Notes. 2016;9(1):485.

26. Haile YG, Alemu SM, Habtewold TD. Common mental disorder and its association with academic performance among Debre Berhan University students, Ethiopia. Int J Ment Heal Syst. 2017:11:34.

27. Moher D, Shamseer L, Clarke M, Ghersi D, Liberati A, Petticrew M, Shekelle P, Stewart LA. Preferred reporting items for systematic review and metaanalysis protocols (PRISMA-P) 2015 statement. Syst Rev. 2015;4:1.

28. The Newcastle-Ottawa Scale adapted for cross-sectional studies [https:// wellcomeopenresearch.s3.amazonaws.com/supplementary/13880/ea30a2fba15a-44a9-b35e-5f0914db80b3.docx]. Accessed 1 Feb 2019.

29. Berkey CS, Hoaglin DC, Mosteller F, Colditz GA. A random-effects regression model for meta-analysis. Stat Med. 1995;14(4):395-411.

30. Doi SA, Thalib L. A quality-effects model for meta-analysis. Epidemiology (Cambridge, Mass). 2008;19(1):94-100

31. Higgins JP, Thompson SG. Quantifying heterogeneity in a meta-analysis. Stat Med. 2002;21(11):1539-58.

32. Liu JL. The role of the funnel plot in detecting publication and related biases in meta-analysis. Evid Based Dent. 2011;12(4):121-2.

Ready to submit your research? Choose BMC and benefit from:

- fast, convenient online submission

- thorough peer review by experienced researchers in your field

- rapid publication on acceptance

- support for research data, including large and complex data types

- gold Open Access which fosters wider collaboration and increased citations

- maximum visibility for your research: over $100 \mathrm{M}$ website views per year

At $\mathrm{BMC}$, research is always in progress.

Learn more biomedcentral.com/submissions 